\title{
Title: Miswired enhancer logic drives translocation positive
}

\section{rhabdomyosarcoma}

running title: Miswired enhancer logic drives rhabdomyosarcoma

keywords: 3D Chromatin, Enhancer Logic, Childhood Cancer, Transcription Factors

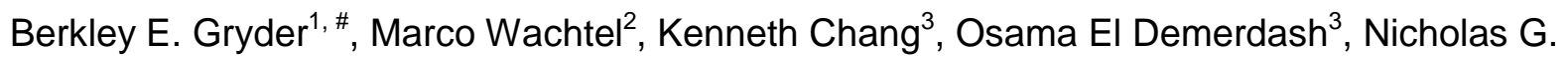

Aboreden ${ }^{1}$, Wardah Mohammed ${ }^{1}$, Winston Ewert ${ }^{4}$, Silvia Pomella ${ }^{5}$, Rossella Rota ${ }^{5}$, Jun S. Wei ${ }^{1}$,

Young Song ${ }^{1}$, Beat Schaefer ${ }^{2}$, Christopher R. Vakoc ${ }^{3}$, and Javed Khan ${ }^{1, \#}$

1- Genetics Branch, National Cancer Institute, NIH, Bethesda, MD 20892, USA

2- University Children's Hospital, Zurich, Switzerland

3- Cold Spring Harbor Laboratory, 1 Bungtown Road, Cold Spring Harbor, NY 11724, USA

4- Biologic Institute, Redmond, Washington, USA

5- Department of Oncohematology, Ospedale Pediatrico Bambino Gesu' Research Institute, IRCCS,

Rome, Italy

${ }^{\#}$ Corresponding author

\section{Correspondence:}

Javed Khan (khanjav@mail.nih.gov)

Berkley Gryder (berkley.gryder@nih.gov) 


\section{Abstract}

Core regularity transcription factors (CR TFs) define cell identity and lineage through an exquisitely precise and logical order during embryogenesis and development. These CR TFs regulate one another in three-dimensional space via distal enhancers that serve as logic gates embedded in their TF recognition sequences. Aberrant chromatin organization resulting in miswired circuitry of enhancer logic is a newly recognized feature in many cancers. Here, we report that $P A X 3-F O X O 1$ expression is driven by a translocated FOXO1 distal super enhancer (SE). Using 4C-seq, a technique detecting all genomic regions that interact with the translocated FOXO1 SE, we demonstrate its physical interaction with the PAX3 promotor only in the presence of the oncogenic translocation. Furthermore, RNA-seq and ChIP-seq in tumors bearing rare PAX translocations implicate enhancer miswiring is a pervasive feature across all FP-RMS tumors. HiChIP of enhancer mark H3K27ac showed extended connectivity between the distal FOXO1 SE and additional intra-domain enhancers and the PAX3 promoter. We show by CRISPR-paired-ChIP-Rx that PAX3-FOXO1 transcription is diminished when this network of enhancers is selectively ablated. Therefore, our data reveal a mechanism of a translocated hijacked enhancer which disrupts the normal CR TF logic during skeletal muscle development (PAX3 to MYOD to MYOG), replacing it with an infinite loop logic that locks rhabdomyosarcoma cells in an undifferentiated proliferating stage. 


\section{Introduction}

Control of the expression of the core regulatory transcription factors (CR TFs) that guide developmental decision making are directed by logical enhancer elements ${ }^{1-3}$. These genomic elements, when heavily activated, become super enhancers (SEs) with unusually large deposits of active histone marks, chromatin regulators and transcriptional coactivators ${ }^{4}$. Chromosomal rearrangements allowing SEs to drive oncogene expression is an emerging mechanism in tumor biology ${ }^{5,6}$. Alveolar (fusion-positive) rhabdomyosarcoma (FP-RMS), an aggressive skeletal muscle cancer of childhood, often possesses chromosomal translocations, involving commonly PAX3 and FOXO1 genes, rarely PAX7-FOXO1, and in exceptional cases PAX3-INO80D and PAX3-NCOA1 fusions ${ }^{7}$. Disruption of CR TF transcription is effectual as FP-RMS treatment ${ }^{8,9}$. During normal skeletal muscle development, PAX3 initiates specification of the muscle lineage and is shut off during myogenic differentiation. Consequently master regulators MYOD and finally MYOG promote muscle progenitor cells to exit cell division and complete muscle differentiation ${ }^{10}$. However, although FP-RMS cells express these master regulators needed to trigger muscle differentiation program, they are halted in an early myoblastic and thus more proliferative state and not able to complete cell differentiation. Fusion gene product resulted from the translocation has been thought to be responsible of the inability of differentiation for the FP-RMS. However, the mechanism of how the oncogenic fusions lock FP-RMS cells in their myoblast state has not been fully understood. In this study, we test the hypothesis that the chromosomal translocation event resulted in novel enhancer/promoter interactions to maintain robust expression of the oncogenic fusion protein in FP-RMS.

When conducting the first epigenetic landscape study of FP-RMS we uncovered a strong dependence on SEs for tumor survival, with PAX3-FOXO1 being a chief determinant of SE formation in collaboration with MYOD and MYOG, and oncogene $\mathrm{MYCN}^{9}$. Using chromatin 
conformation capture (3C, 4C-seq) and chromatin immunoprecipitation (ChIP-seq, ChIP-Rx, HiChIP) based assays, we here study a key SE $300 \mathrm{~kb}$ distal of FOXO1 which is occupied by all four of these master regulators, and examine its function in FP-RMS. We propose that hijacking SEs bound by myogenic CR TFs allows for continued expression of oncogenic PAX fusions, thus circumventing normal myogenic enhancer logic.

\section{Results}

\section{Chromosomal translocation imports the FOXO1 super enhancer to the PAX3 promoter}

Precisely how PAX3-FOXO1 locks the cells into a myoblastic state unable to differentiate is unknown. Proper enhancer-promoter interactions are enabled by constraints in 3D chromatin folding, determined by CTCF and cohesion formed loops at topologically associated domain (TAD) boundaries ${ }^{11,12}$. PAX3 is normally silenced during progression past the myoblast stage of muscle differentiation. PAX3 expression during embryogenesis is tightly controlled, and structural variation that disrupts the PAX3 TAD causes limb malformation ${ }^{13}$. We hypothesized that the fusion event results in novel enhancer/promoter looping events to maintain fusion protein expression independent of normal lineage control. Hi-C data ${ }^{14}$ indicated 3 candidate topological loops containing wild-type FOXO1 that exist in normal cells. We found all of these were occupied by RAD21 (of the cohesion complex) and CTCF in FP-RMS RH4 cells by ChIPseq (Fig. 1a). CTCF binding events that form loops most often have binding motif sequences that are antiparallel (and point inward toward each other) ${ }^{14}$. The CTCF motif orientation at the $1^{\text {st }}$ and $3^{\text {rd }}$ of these sites near FOXO1 were found to be antiparallel with the CTCF motif near the PAX3 promoter, permissive of chromatin loop formation via extrusion after the translocation.

To identify interacting domains cis to the $P A X 3$ promoter after the translocation, we used circularized chromatin conformation capture followed by sequencing (4C-seq) from viewpoint anchors around the PAX3 promoter and FOXO1 genes on chromosomes 2 and 13. 
Remarkably, looping was detected between the PAX3 promoter and multiple candidate enhancers downstream of FOXO1, and was restricted between the intronic fusion breakpoint in FOXO1 and the predicted topological boundary (Fig. 1a). The outermost TAD-boundary looping interaction was confirmed by Sanger sequencing of the 3C PCR product (Extended Data Fig. 1a-c). Notably, each of the 3 CTCF sites 3' of FOXO1 formed looping interactions with PAX3 only in translocation-positive $\mathrm{RH} 4$, but not in the translocation-negative RMS cell line CTR (Extended Data Fig. 1d). A previous study of 4C-seq in FP-RMS cell lines has shown similar interactions consistent with our results ${ }^{15}$, but here we provide the first ChIP-seq informed functional interpretation of these interactions as CTCF bounded enhancers that contain critical CR TFs. We hypothesize these newly juxtaposed enhancer elements keep the PAX3 promotor on the translocated allele active. These enhancers could sustain the continual PAX3-FOXO1 expression and the oncogenic process because they harbor strong binding sites for MYOD1, MYCN and MYOG (Figure 1b-c). 


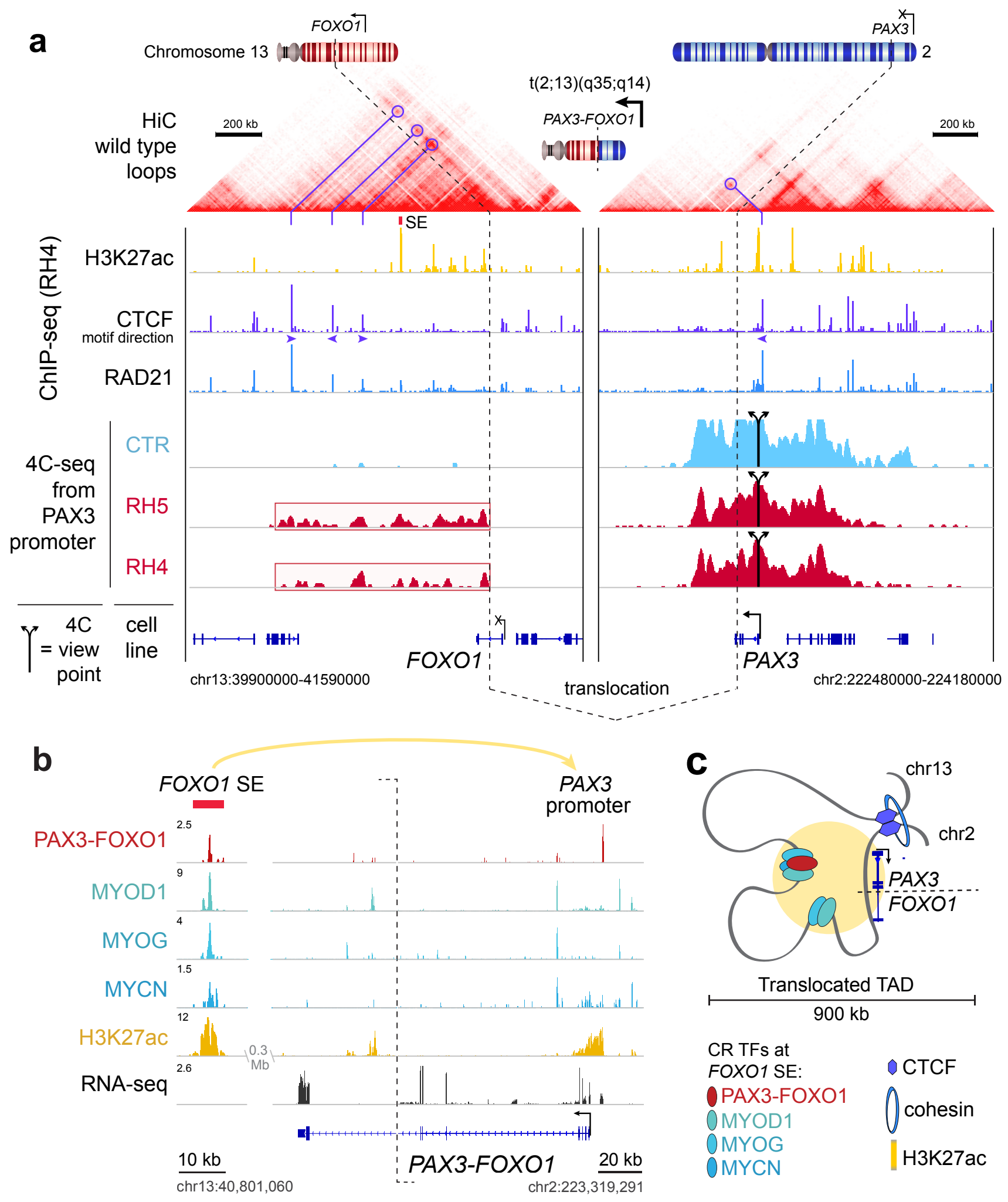

Figure 1. Translocation restructures insulated neighborhood surrounding PAX3-FOXO1

a. Wild-type loops indicated by Hi-C profile from human GM12878 cells. ChIP-seq demonstrates binding locations of H3K27ac, CTCF and RAD21 in RH4 cells. 4C-seq reveals looping between 
viewpoints at CTCF sites bounding FOXO1 enhancers, and the PAX3 promoter, in translocation negative (CTR) and translocation positive (RH5, RH4) cells. Viewpoints are indicated by split arrows, and translocation breakpoints are indicated by dotted lines.

b. ChIP-seq signal for Master Transcription Factors and H3K27ac, and RNA-seq signal, in Reads Per Million (RPM), at the FOXO1 Super Enhancer (SE) and PAX3-FOXO1 fusion gene. c. Schematic of translocation created a new Topologically Associated Domain (TAD) bringing the PAX3-promoter (chr2) under the control of FOXO1 SE and other smaller enhancers (chr13).

\section{Rare PAX-fusions implicate enhancer miswiring}

Besides the PAX3-FOXO1 translocation, there are several other PAX translocation variations in FP-RMS including PAX7-FOXO1, PAX3-NCOA1, and PAX3-INO80D (Figure 2a). While there is protein homology between PAX3 and PAX7 (with similar DNA binding domains), NCOA1 or INO80D share hardly any protein homology with the FOXO1 transactivation domain. However, RNA-seq reveals that remarkably similar transcriptome profiles from tumors harboring these diverse oncogenic fusions ${ }^{7}$. Additionally, SEs (found in $\mathrm{RH} 4$ cells to be bound by FP-RMS specific CR TFs) exist near all known translocation partner genes (Figure 2b). This led us to hypothesize that enhancer miswiring as a result of chromosomal translocations may be the common theme among all PAX-fusions tumors.

In FP-RMS with a PAX3-FOXO1 translocation, if only the promoter of $P A X 3$ determines the expression of the PAX3 gene on the wild-type allele and PAX3-FOXO1 fusion gene on the translocated allele, the expression of $P A X 3$ exons will be even. The expression of $P A X 3$ exons of will be uneven if PAX3-FOXO1 is regulated by the abnormal juxtaposition of the FOXO1 SE, since the last exons of $P A X 3$ are not influenced by the FOXO1 SE (both from the remaining wildtype $P A X 3$ and the reciprocal FOXO1-PAX3 translocated allele). Therefore, we examined 
exon level expression of the genes involved in translocation using RNA-seq data from FP-RMS patient tumors. The RNA-seq data showed that exons before the translocation (3-prime or Nterminal) are always expressed significantly higher than those beyond the translocation breakpoint (5-prime or C-terminal) (Figure 2c). For example, exon-level expression in tumors with $P A X 3-F O X 01$ revealed that the final two post breakpoint exons of $P A X 3$ were greatly under expressed only in the tumors with PAX3 fusion genes, indicating that only the exons involved in the fusion event is expressed due to activation by the FOXO1 SE. On the other hand, exon usage of a gene is balanced in RMS patients lacking the translocation, e.g. PAX3 in fusion negative RMS (Figure 2c). Importantly, inferring allele selective expression via RNA-seq allows interrogation of extremely rare PAX fusions, PAX3-INO80D and PAX3-NCOA1. All fusion gene partners showed exonic imbalance resulting from favored expression of the translocated exons (Fig 2c, Extended Data Fig 2).

ChIP-seq data in FP-RMS cell lines and patients allowed us to discover recurrent SEs surrounding not only FOXO1, but also rare partner NCOA1 (Figure 2d). Although SEs represent only $\sim 4 \%$ of enhancers, their unique and consistent presence argues that these diverse fusions may uniformly rewire SEs which can be active in the epigenomic state of all FP-RMS patients. Here we report the first epigenomic data generated for a PAX3-NCOA1 patient, and we found this rare fusion epigenetically phenocopies tumors with PAX3-FOXO1 or PAX7-FOXO1 (Figure 2d). When juxtaposed, these recurrent SEs are key elements driving the expression of fusions genes. 

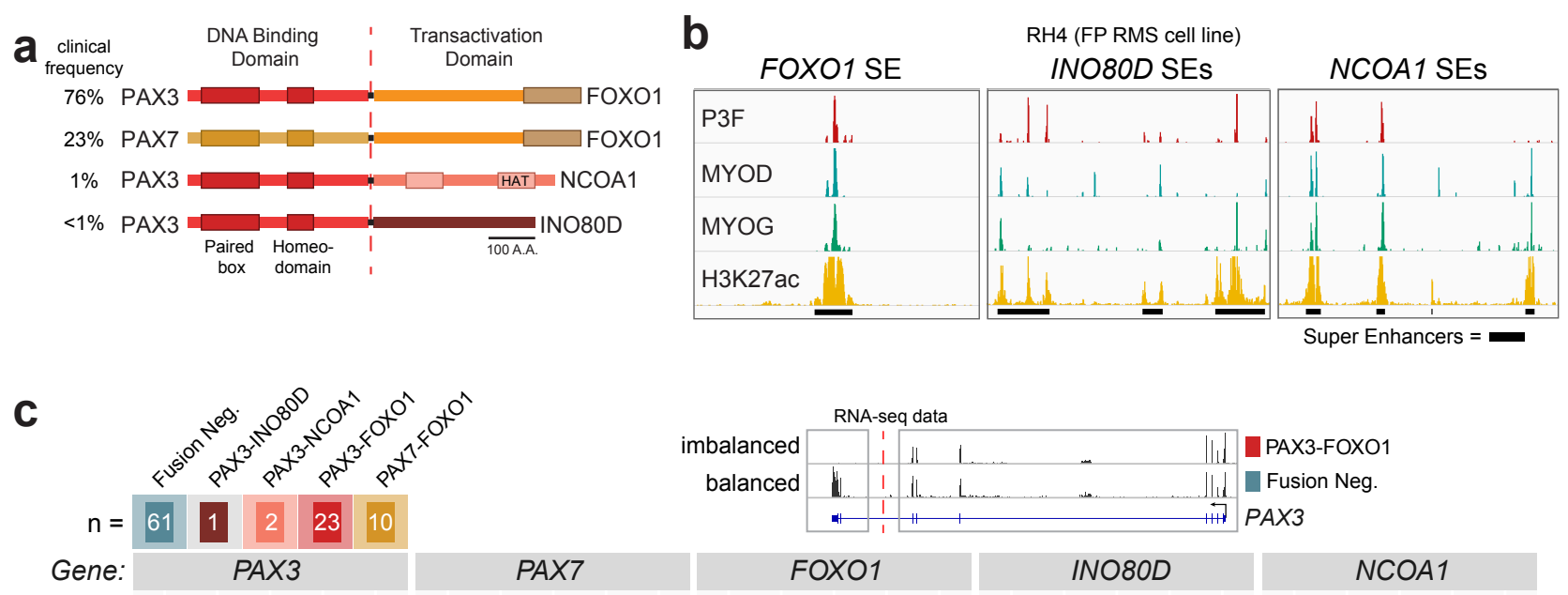

$$
\text { Gene: } \quad P A X 3
$$

PAX7

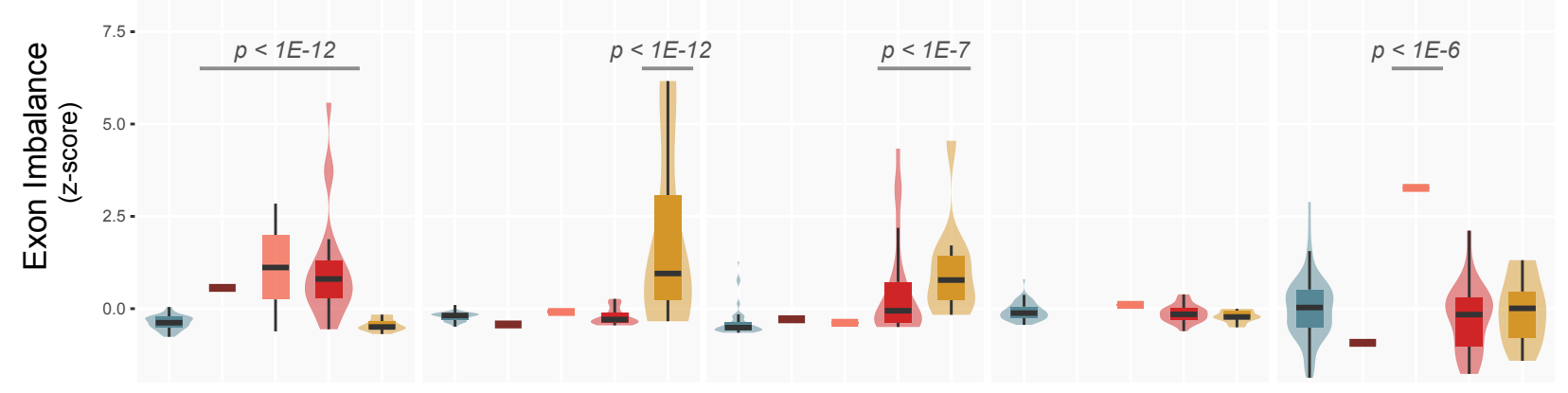

d
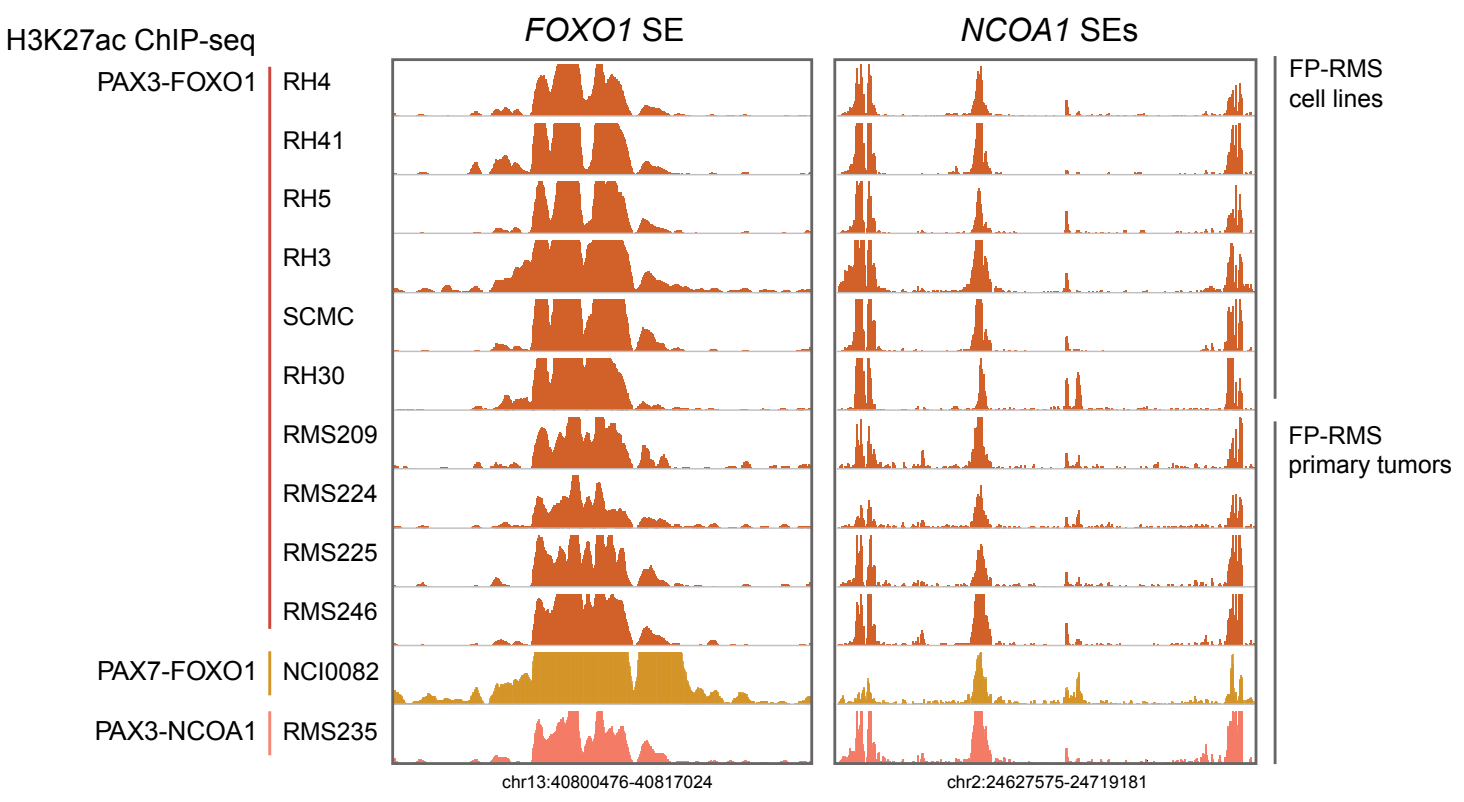

Figure 2. SEs and allele specific expression at rare PAX3 translocation partners

a. PAX fusions and their clinical frequency in FP-RMS patient tumors.

b. SEs in RH4 (PAX3-FOXO1 bearing cells) include not only those near FOXO1, but also

INO80D and NCOA1. 
c. Exonic imbalance measure in RNA-seq data from primary tumors and cell lines of FN-RMS ( $\mathrm{n}$ $=61)$, FP-RMS with PAX3-INO80D fusion $(n=1)$, PAX3-NCOA1 fusion $(n=2)$, PAX3-FOXO1 $(n=23)$ and PAX7-FOXO1 $(n=10)$. P-values were calculated using a two-tailed t-test with Welch's correction.

d. H3K27ac ChIP-seq in FP-RMS cell lines $(n=6)$ and primary tumors $(n=6)$ at the FOXO1 SE and the NCOA1 SES.

\section{CRISPR reveals essentiality of cis-regulatory elements regulating PAX3-FOXO1}

To build on the evidence from $4 \mathrm{C}$, we set out to gain a more complete dataset confirming the interactions between the enhancer network controlling PAX3-FOXO1. Thus, we used HiChIP against H3K27ac to capture protein-directed interaction frequency between acetylated chromatin sites at enhancers and promoters ${ }^{16}$. The results identified that the FOXO1 super enhancer is connected not only to the $P A X 3$ promoter, but also to three smaller intergenic and intronic enhancer elements near or within FOXO1 (Figure 3a-b).

We next measured the contribution of these enhancer elements to the overall survival of FP-RMS cells. We designed a library of sgRNAs against each enhancer or promoter constituent, each DNase-hyper sensitive site and each CTCF peak as defined by genome-wide profiles in $\mathrm{RH} 4$ cells. We introduced them in a pooled fashion by viral infection into $\mathrm{RH} 4$ cells expressing Cas9. The abundance of each sgRNA in the population was then quantified over time using next-generation sequencing (at day 2, 5, 7, 10, 13, 17, 20 and 25). sgRNA's that target the PAX3 promoter had the strongest impact on $\mathrm{RH} 4$ cell viability, as inferred from the largest reduction in guide representation over time (Figure 3c). Among CTCF sites, two candidate anchor sites (FOXO1-distal sites \#2 and \#3) had no negative influence, while the outermost CTCF sites (FOXO1-TAD boundary site \#1 and PAX3-TAD boundary) were both reduced by negative selection (Extended Data Figure 3). Among TF-bound and H3K27ac 
decorated enhancers, sgRNA's targeting SEs within the TAD (the translocation-induced insulated neighborhood containing PAX3-FOXO1) were more effective than sgRNA's targeting SEs outside this neighborhood, and also more than typical enhancers (Figure 3d).

Individual sgRNA's were next used to study the impact on PAX3-FOXO1 transcription. We confirmed our hypothesis that disruption of the FOXO1 SE reduced PAX3-FOXO1 at the transcript level and protein level at 24 hours after sgRNA infection (Figure 3e-f). To attribute the effect of this sgRNA to direct impairment of the enhancer, we assayed H3K27ac changes by ChIP-Rx (spike in reference normalized ChIP-seq) ${ }^{17}$. The results revealed that the FOXO1 SE was not only depleted of H3K27ac, but that the associated enhancer interaction network (as HiChIP identified) was also drastically reduced of acetylation at the sites interacting with FOXO1 SE (Figure 3g). Conversely, this enhancer network was not impaired by a sgRNA targeting the first exon of $P A X 3$, except for slight reduction in acetylation levels at the $P A X 3$ promoter (Figure $3 \mathrm{~g}$ ). These data demonstrated that the FOXO1 SE is essential to maintain the expression of PAX3-FOXO1 oncogene in RMS. 

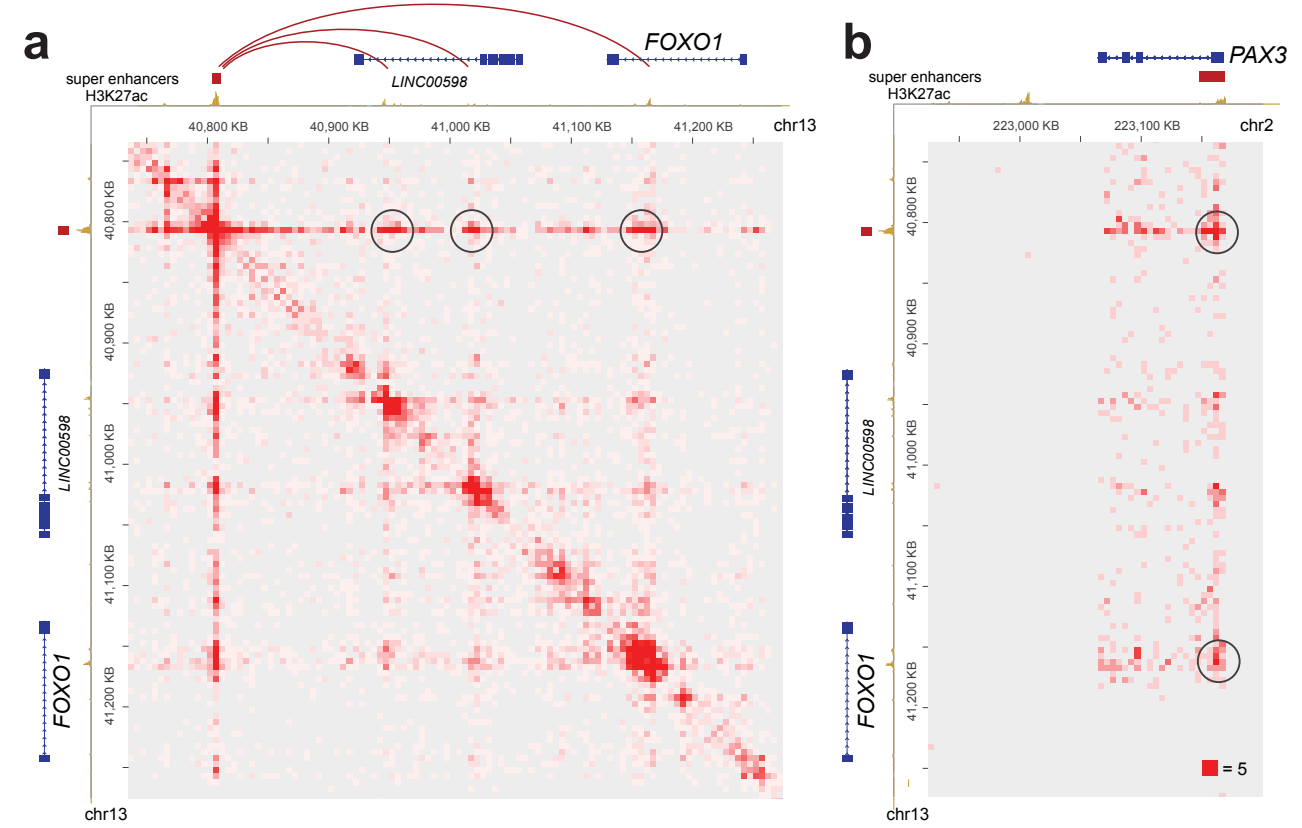

C
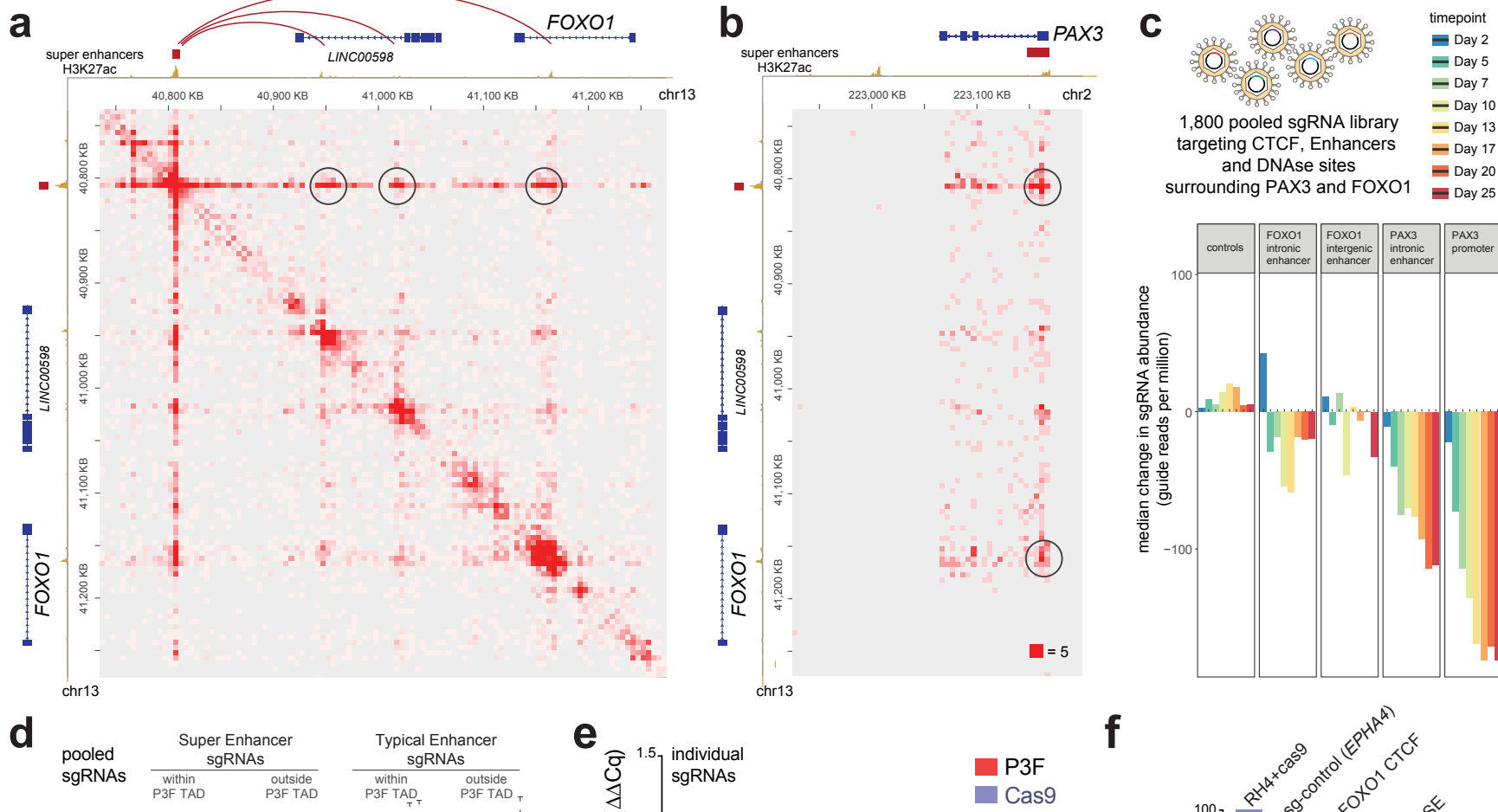

e
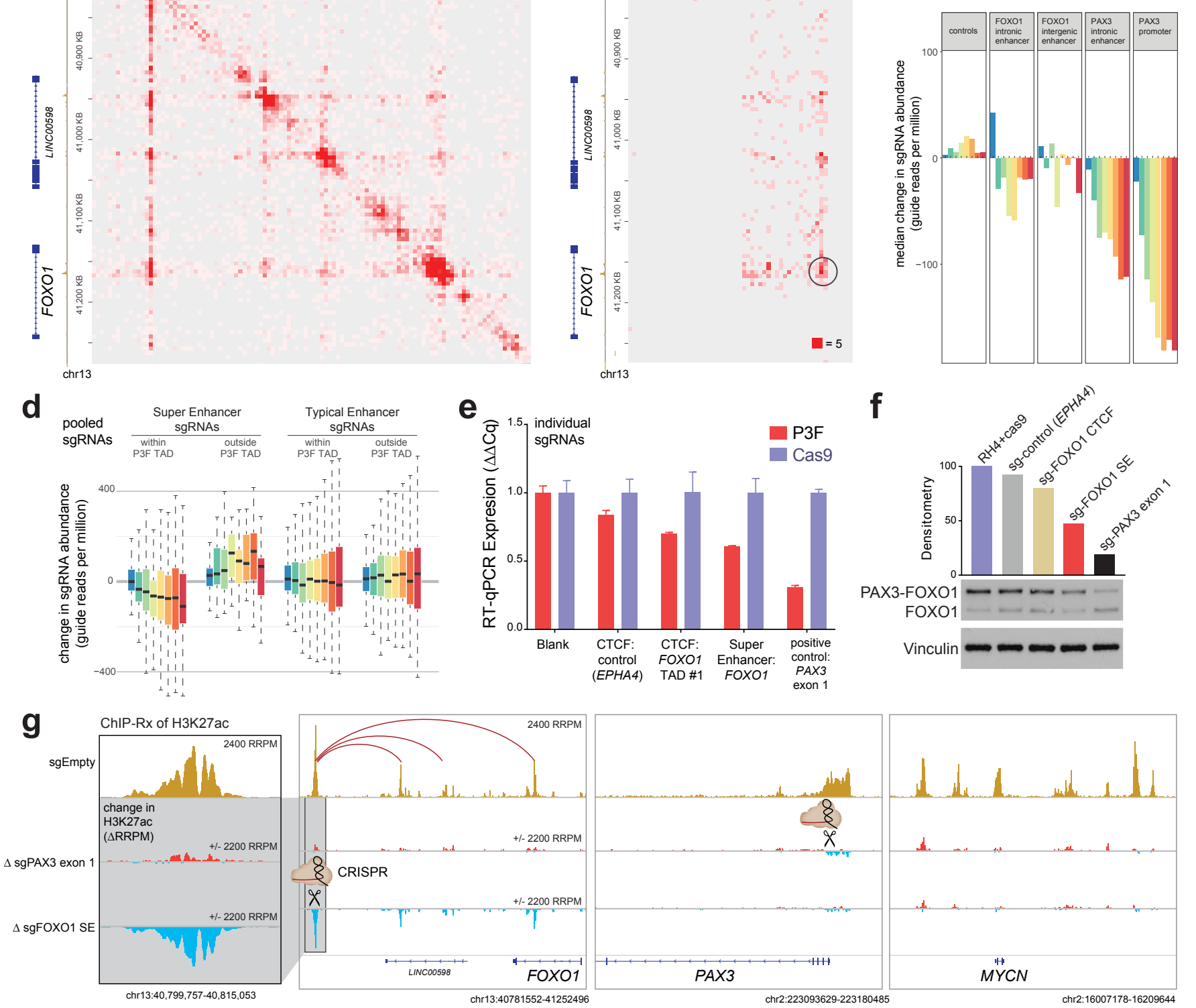

Figure 3. CRISPR functional mapping of non-coding elements controlling PAX3-FOXO1

a. H3K27ac HiChIP reveals structure of FOXO1 SE interactions with smaller intra-TAD enhancer elements.

b. Interaction by H3K27ac HiChIP between PAX3 promoter and FOXO1 SE and intronic enhancer element. 
c. Pooled sgRNA CRISPR screening tiling against cis-regulatory genomic elements surrounding PAX3 and FOXO1 defined their degree of essentiality. $\mathrm{RH} 4$ cells expressing Cas 9 were sampled by counting sgRNA abundance using sequencing at the indicated time intervals.

d. Change in sgRNA abundance of pooled CRISPR shows intra-TAD super enhancers are more critical for $\mathrm{RH} 4$ cell survival than typical enhancers, or SEs outside TAD boundaries.

e. Individual sgRNA impact on PAX3-FOXO1 gene expression after 1 day of transduction in FPRMS cells RH4 expressing cas9.

f. PAX3-FOXO1 protein levels are reduced by individual sgRNAs targeting key cis-regulatory elements, especially those targeting the FOXO1 SE and the first exon of PAX3.

g. ChIP-seq with reference exogenous spike-in (ChIP-Rx) for H3K27ac was employed to interrogate the chromatin impact of the sgRNA targeting the FOXO1 SE. The top track is control ChIP-Rx (sgEmpty), the second track is showing the change (delta RRPM) upon CRISPR of the $1^{\text {st }}$ exon of PAX3, and the third track shows the change in H3K27ac upon CRISPR of the FOXO1 SE at 24 hours post sgRNA transduction. All experiments were performed in $\mathrm{RH} 4$ cancer cells.

\section{FOX01 SE is activated during a key step in myogenesis}

To examine if the activity of the FOXO1 SE was coordinated with myogenic steps, we utilized ENCODE data mapping H3K27ac in various stages of the muscle lineage. We found that the FOXO1 enhancer is transiently transformed into a super enhancer during myogenesis at the same time MYOG acquires a super enhancer (Figure 4a). FOXO1, MYOG, and MYOD1 have more highly ranked SEs in FP-RMS as compared to FN-RMS (Figure 4b), in agreement with the notion that FP-status is more advance toward myotubes, and FN-status is more similar the earlier myoblast state. MYOG activation is commonly prevented by mutant-RAS signaling 
through MEK/ERK in FN-RMS tumors, which can be rapidly release via small molecule inhibitors of MEK/ERK ${ }^{18}$. Using this system, we asked if the FOXO1 SE and concomitant FOXO1 expression was induced alongside MYOG activation. Indeed, not only did we find FOXO1 to be upregulated, but also observed MYOG invasion on the same SE which is recruited during the PAX3-FOXO1 translocation event in FP-RMS (Extended Data Fig. 4a).

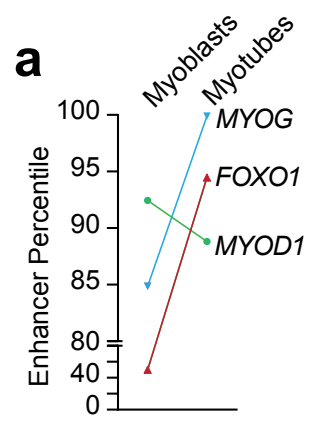

b FOXO1 Enhancer

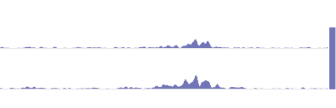

SE
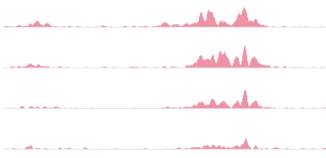

d

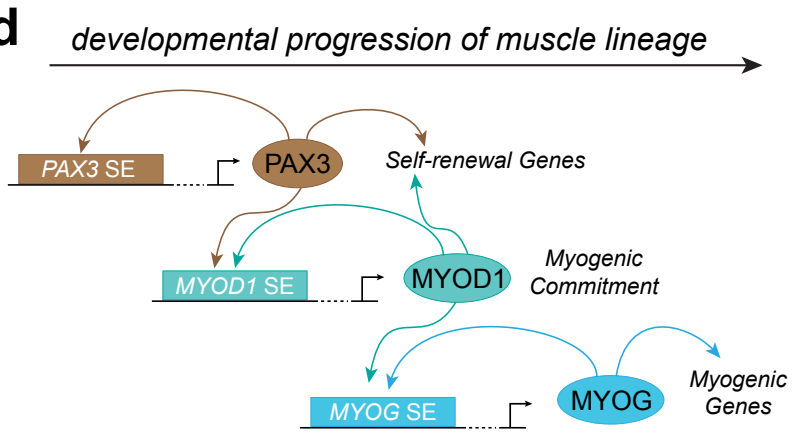

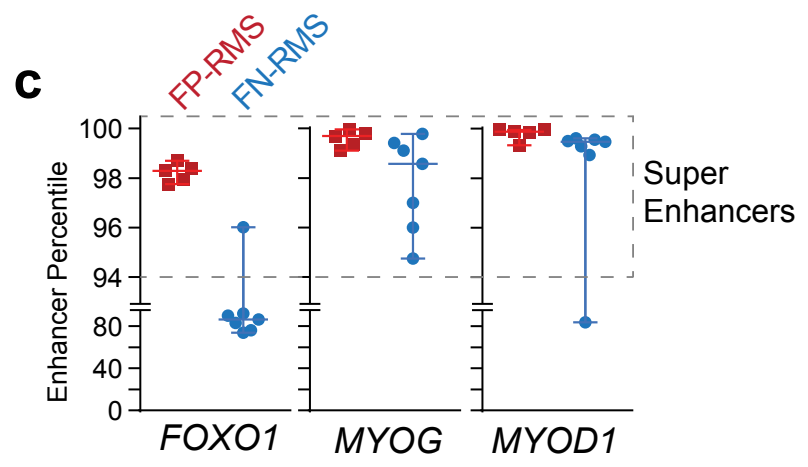

e

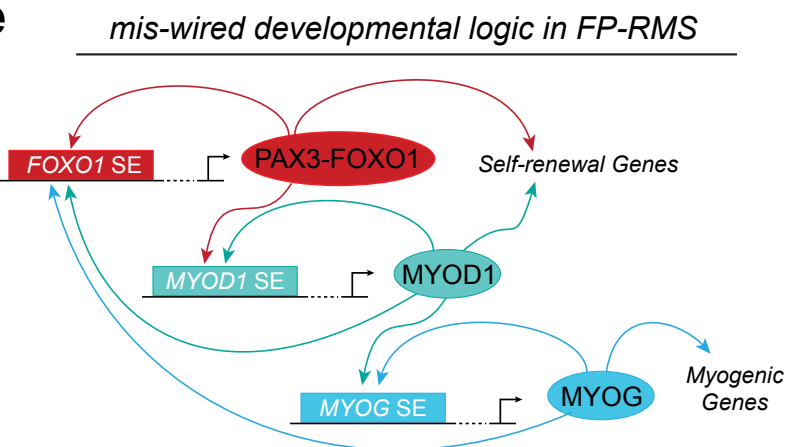

Figure 4. Miswired super enhancer logic able to maintain an oncogenic cell state

a. Increased FOXO1 and MYOG enhancer rank (by percentile) in the transition from myoblasts to myotubes.

b. Enhancer rank of FOXO1, MYOG and MYOD1 in RMS, as measured by rank of H3K27ac ChIP-seq bound to each enhancer.

c. Model illustrating normal development of the muscle lineage.

d. Miswiring of myogenic circuitry due to translocation of FOXO1 SE to PAX3 promoter, allowing MYOD and MYOG to activate PAX3-FOXO1. 


\section{Discussion}

Two factors can be selected for in rearrangement driven cancers: oncogenic biochemical function in the case of a resulting fusion $\operatorname{protein}^{19}$, or aberrant expression levels of a protooncogene (such as MYC or GFI1) via enhancer hijacking ${ }^{5}$. In FP-RMS, PAX-fusions are selected for both an oncogenic fusion protein product and miswired enhancer logic, effectively reprograming core regulatory TF networks.

PAX3 activates MYOD via binding and activating MYOD1 SEs, but then shuts off presumably because MYOD does not work backwards to upregulate PAX3 (MYOD ChIP-seq shows no binding in the PAX3 promoter, unlike the MYOG promoter, Extended Data Fig. 4b). Lacking enhancers responsive to MYOD/MYOG, the remaining wild-type alleles of $P A X 3 / 7$ in FP-RMS tumors are silent. Our data suggests that newly juxtaposed enhancer elements initiate and continually drive PAX3-FOXO1 expression, implicating that enhancer miswiring is at the heart of the oncogenic process in FP-RMS. When the FOXO1 SE is translocated to regulate $P A X 3$, late myogenic factors (MYOG/MYOD) work through this SE to drive an early myogenic factor (PAX3), changing a "progressive" enhancer logic into an "infinite loop" enhancer logic. Similarly, CR TF logic circuits are self-reinforcing in self-renewal and proliferative states such as embryonic stem cells ${ }^{20}$.

Analysis of RNA-seq for patients with non-FOXO1 partners (INO80D, NCOA1) of PAX3 reveals a remarkably similar transcriptome ${ }^{7}$, despite not being TFs themselves, and having no sequence homology to the activation domain (AD) of FOXO1. It has been shown that TFs can perform their function even when their ADs are swapped with those of other TFs ${ }^{21}$. This tolerance to diverse $A D$ sequence may be explained by the fact they share the common property of being intrinsically disordered, a feature needed to support phase-separation capacity of TFs ${ }^{22}$. Indeed, the portions of FOXO1, INO80D and NCOA1 involved in PAX3 fusion 
oncoproteins are predicted to be heavily disordered (Extended Data Figure 5a-c). Remarkably, although transcription factors as a class are heavily disordered (Extended Data Figure 5d), yet PAX fusion partners are particularly disordered (similar to FET family fusions like EWSR1). A related partner MAML3 is almost entirely disordered (Extended Data Figure 5e) and PAX3MAML3 fusions occur in biphenotypic sinonasal sarcoma (SNS) but not in $\mathrm{RMS}^{23}$. MAML3 lacks a SE in myogenesis or RMS, and our model would therefore predict the absence of PAX3MAML3 translocations in RMS. SNS may arise from a cell of origin whose epigenome has a lineage restricted enhancer at MAML3 which gets recruited in SNS tumorigenesis. Future studies could reveal phase condensate formation as a common capability of all PAX3 fusions found in these tumors.

Many disordered proteins in the genome are not involved in PAX3 fusions. A parallel criterion for a successfully tumorigenic fusions could be the presence of an active SE in the same lineage step as PAX3 (such as those SEs proximal to FOXO1, INO80D and NCOA1 in the RMS-specific epigenetic state). Super enhancer-containing loci may be enriched in translocations for two reasons. First, active enhancers are transcriptionally active and early replicating ${ }^{24}$, and thus likely more susceptible to double strand breakage ${ }^{25}$. Secondly, among translocations which form, those resulting in overexpression of an oncogene are selected for, and SEs can enable such continued overexpression. The appearance of certain SEs is transient and logically restricted to certain points in development, and thus may be restricting the potential miswiring events that could give rise to an "infinite loop" in CR TF logic. We propose this can explain, at least in part, the selection of translocation partners in FP-RMS tumors, and provides a paradigm likely relevant to other translocation driven cancers. 


\section{Methods}

\section{Circularized chromatin conformation capture (4C-seq)}

4C-seq was performed on RH4, RH5 and CTR cells as previously described ${ }^{9}$. Briefly, cells were grown in DMEM at $37^{\circ} \mathrm{C}$, then chemically crosslinked with $1 \%$ formaldehyde for 12 minutes. Then, in-situ digestion and in-situ re-ligation of 3D contacts (keeping nuclei intact) was performed using Dpnll as the 4-bp DNA cutter. Following re-ligation, Csp6I was used to reduce template size, followed by relegation to circularize for inverse PCR at viewpoints of interest surrounding $P A X 3$ and FOXO1. 4C samples of RMS cell lines were amplified using bait region primers for PAX3-promoter (F: CAAGGAGTCCTGGTGCCAAA, R:

CACTGGCCGGTGAGAAGG). We also studied three CTCF sites on the FOXO1 side with the following primers: FOXO1-CTCF.1 (F: GCTCCCACAGAAGAAGCAGA, R:

GGTGGAGACAGAGGCAGTAC), FOXO1-CTCF.2 (F: CACACACAAGCAAGCACAGA, R: AGCCTCATTACCACTTTTGAACA), and FOXO1-CTCF.3 (F: TCAGGAAGGTTCAAACTTACTTTCC, R: CACGCACGCATAAAAGAGCA). Illumina TruSeq ChIP Library Prep Kit was used on purified inverse PCR products, and sequenced (75 bp single end) with an Illumina NextSeq500 (and, are typically multiplexed with ChIP-seq experiments to increase sequence diversity, which is needed because most reads from a single $4 \mathrm{C}$ viewpoint are identical duplicated products from self-ligation).

\section{C-seq data analysis}

Reads from $4 \mathrm{C}$ experiments were first filtered to keep only reads containing the bait primer sequence (from the viewpoint), tolerating 1 mismatched base pair. Then, barcodes and viewpoint sequence were trimmed, followed by mapping to hg19 with bwa. Reads surrounding the viewpoint (within $4 \mathrm{~kb}$ ) were removed to aid visualization of informative distal contacts. Smoothing was performed by averaging over a sliding window of $5 \mathrm{~kb}$ and visualized in IGV. 


\section{C confirmation PCR and sequencing}

Primers were designed to validate interaction between the outermost CTCF boundaries surrounding $P A X 3$ and FOXO1 (Extended Data Figure 1). The left primer overlapped a digestion site detected to form a long-range ligation at high frequency in $4 \mathrm{C}$ data, with the sequence: AAGCAGATGGGGATCACGTG (Dpnll cutsite in bold). The right primer was designed close to the PAX3 CTCF site, but before any additional cutsites:

GCAGCCAGTGAGATAAGACATTA. PCR was performed using Phusion High Fidelity Master Mix (NEB) with $30 \mathrm{pmol}$ of each primer and $70 \mathrm{ng}$ of $3 \mathrm{C}$ DNA template (same template from which $4 \mathrm{C}$ was prepared, prior to the second Csp6l cut), with 25 cycles ( $94^{\circ} \mathrm{C}$ for 15 seconds, 55 ${ }^{\circ} \mathrm{C}$ for 30 seconds, $70^{\circ} \mathrm{C}$ for 30 seconds) with completed with 1 minute at $70{ }^{\circ} \mathrm{C}$. PCR product was purified with PCR QiaQuick kit (Qiagen), then Sanger sequencing was performed at the Center for Cancer Research Sequencing Facility (CCR-SF).

\section{Exonic imbalance analysis of RMS RNA-seq}

RNA-seq read counts were generating using cufflinks at the exon level for each sample (RMS primary tumor data $)^{7}$. Then, for each RMS tumor $(n=97)$, the exonic balance before and after the known break points were calculated (average FPKM of pre-breakpoint exons/average FPKM of post-breakpoint exons) for all known translocation partners (PAX3, PAX7, FOXO1, NCOA1, INO80D). Normal exonic bias was corrected for by taking a z-score across all samples (including FN-RMS samples). Plots of exon imbalance where made in R using ggplot2.

\section{$\mathrm{HiC}$ and HiChIP data analysis}

$\mathrm{HiC}$ data for high-resolution contact frequencies in $\mathrm{GM} 12878$ cells $^{14}$ was downloaded and visualized through the Juicebox desktop application ${ }^{26}$. Candidate native loop contacts (purple circles, Figure 1) were annotated manually from visual inspection, and CTCF motif orientation was derived by taking the intersect of CTCF peaks in $\mathrm{RH} 4$ cells called with MACS2 (previously 
published, GEO accession number GSM2214099) and CTCF recognition sequences annotated in the HOMER known motifs collection (http://homer.ucsd.edu/homer/motif/genomeWideMotifScan.html).

H3K27ac HiChIP data analysis of data generated in $\mathrm{RH} 4$ cells was performed using the Hi-Pro pipeline $^{27}$. We kept only high-quality paired-end tags (PETs), using the HiC-Pro pipeline to filter out reads lacking the restriction site (DpnlI), filter out duplicates, and filter out poorly mapped reads. Valid contact pairs remaining were converted to .hic format using the hicpro2juicebox.sh script (https://github.com/nservant/HiC-Pro/tree/master/bin/utils), to enable compatibility with the Juicebox visualization tool.

\section{Lentivirus production}

A lentivirus plasmid mix was made by combining Lenti-Rev, Lenti-PM2, Lenti-Tat and Lenti-VsvG plasmids at a ratio of 1:1:1:2. HEK293T cells were seeded at 50-60\% confluency (2 million cells) in a $10 \mathrm{~cm}$ dish in $10 \mathrm{~mL}$ media (DMEM, 10\% FBS) and incubated at $37^{\circ} \mathrm{C}, 5 \% \mathrm{CO}_{2}$. The next day, $5 \mu \mathrm{g}$ of transfer plasmid, $10 \mu \mathrm{g}$ of lentivirus plasmid mix and $36 \mu \mathrm{L}$ of $\mathrm{X}$-tremeGENE HP DNA transfection reagent (Sigma) were mixed into $1.2 \mathrm{~mL}$ serum-free OptiMEM (Gibco), vortexted and incubated for 15 minutes at RT and added to the cells. Media was changed 24 hours after transfection. Viral supernatant was harvested the following three days and filtered through a $0.45 \mu \mathrm{m}$ syringe filter. Each collection was diluted with 5X PEG-it ${ }^{\mathrm{TM}}$ Virus Precipitation Solution (System Biosciences) and stored at $4^{\circ} \mathrm{C}$ until the final collection. After the final collection, viral supernatants were centrifuged at $1500 \mathrm{~g}$ for 30 minutes at $4^{\circ} \mathrm{C}$. Viral pellets were resuspended in PBS, aliquoted and stored at $-80^{\circ} \mathrm{C}$. All transductions were conducted with 8 $\mu \mathrm{g} / \mathrm{mL}$ polybrene.

\section{Pooled CRISPR Library Design and Construction}


Sequences of enhancer regions ( 100 sites) surrounding PAX3-FOXO1, CTCF, and SE peaks were derived such that all possible sgRNA guides for S.pyogenes Cas9 can be designed within those regions. A total of $1830 \mathrm{sgRNAs}$ were produced. Guides were selected based on having an off-target score of 1.0 denoting the maximum possible score for guides not having any offtarget. Oligonucleotides were synthesized as a pool commercially (Twist Biosciences) and then PCR cloned into BsmBI-cut sgRNA expression vector, LRG2.1T, by using gibson assembly. Deep sequencing analysis was performed on illumina platform and verified that all of sgRNA designs were cloned (data not shown).

\section{Pooled CRISPR-Cas9 screening}

The lentiviral sgRNA library was produced as described above. RH4.Cas cells were first transduced with varying concentrations of the pooled lentiviral library to determine the amount needed for a $\mathrm{MOI}$ of $\sim 0.3$. RH4.Cas cells were then seeded in three $15 \mathrm{~cm}$ dishes at 8 million cells per dish. 24 hours later, cells were transduced with the lentiviral library at a low MOI, leading to $\sim 27 \%$ positive cells (as determined by FACS analysis of GFP expression) and ensuring over $1000 x$ representation of the library. 48 hours after transduction, virus-containing media was removed and replaced with fresh media. 40 million cells were collected and pelleted at days $2,5,7,10,13,17,20$ and 25 post-transduction and 12 million cells were plated at each timepoint for subsequent cell sampling. Coverage at cell level was kept above 1000x throughout the screen.

Sequencing libraries were constructed essentially as previously described (Shi et al., 2015). Genomic DNA (gDNA) from cell pellets was extracted using the AllPrep kit (Qiagen). 32 parallel PCR reactions were performed to amplify sgRNA sequences from gDNA harvested at each timepoint. The total volume of each PCR reaction was $50 \mu \mathrm{L}$ containing $400 \mathrm{ng}$ of gDNA template, $0.2 \mu \mathrm{M}$ forward (TCTTGTGGAAAGGACGAAACACCG) and reverse 
Fisher). PCR cycles were: $1 \times\left(98^{\circ} \mathrm{C}, 2 \mathrm{~min}\right) 35 \times\left(98^{\circ} \mathrm{C}, 8 \mathrm{~s}, 67^{\circ} \mathrm{C}, 12 \mathrm{~s} 72^{\circ} \mathrm{C}, 10 \mathrm{~s}\right), 1 \times\left(72^{\circ} \mathrm{C}\right.$,

5min). Parallel PCR products were pooled together and purified with the QiaQuick kit (Qiagen).

After production of sgRNA amplicons from gDNAs by PCR, ends of fragments were repaired using T4 DNA polymerase (NEB), DNA polymerase I large fragment (Klenow) (NEB), and T4 polynucleotide kinase (NEB), followed by addition of 3' A-overhang with Klenow (3'-5'-exo-) (NEB). Amplicons from each sample were ligated with a unique barcode adaptor (pool) and purified using AMPure magnetic beads (Bechman Coulter). Samples were deep sequenced on Illumina platform. Read counts for each sample were ascertained by mapping raw reads to the sgRNA library sequences. Read counts of each sample were normalized to facilitate data analysis.

\section{Construction of individual sgRNA expression plasmids}

sgRNA sequences were designed using the CRISPR design tool from MIT (crispr.mit.edu). Pairs of DNA oligonucleotides encoding the protospacer sequences were annealed together to create double-stranded DNA fragments with 4-bp overhangs. These fragments were ligated into BsmBI digested Shuttle_sg_RFP657 plasmid. Plasmid constructs were confirmed via sanger sequencing using the LKO1_5 primer (GACTATCATATGCTTACCGT) on the U6 promoter.

\section{Data Access}

Newly generated ChIP-seq data from primary RMS tumors has been made publicly available through the Gene Expression Omnibus (https://www.ncbi.nlm.nih.gov/geo/). The GEO accession number is GSE136799. Code is available at https://github.com/GryderArt.

\section{Acknowledgments}

We thank Michael Kuehl and Katherine Masih for thoughtful discussions of the manuscript. We thank the CCR Genomics Core at the National Cancer Institute, NIH, Bethesda, MD for Sanger 
sequencing. This work was supported by the NCI, NIH. Silvia Pomella is a recipient of a Fondazione Veronesi fellowship.

The content of this publication does not necessarily reflect the views or policies of the

Department of Health and Human Services, nor does mention of trade names, commercial products, or organizations imply endorsement by the U.S. Government.

\section{Disclosure Declaration}

The authors declare no conflicts of interest. 


\section{References}

1 2005, Cell, Boyer, L. A. et al. Core Transcriptional Regulatory Circuitry in Human Embryonic Stem Cells

2 2008, Cell, Chen, X. et al. Integration of External Signaling Pathways with the Core Transcriptional Network in Embryonic Stem Cells

3 2013, Cell, Lee, Tong I. \& Young, Richard A. Transcriptional Regulation and Its Misregulation in Disease

4 2013, Cell, Hnisz, D. et al. Super-Enhancers in the Control of Cell Identity and Disease

5 2014, Nature, Northcott, P. A. et al. Enhancer hijacking activates GFI1 family oncogenes in medulloblastoma

6 2016, Nature genetics, Bandopadhayay, P. et al. MYB-QKI rearrangements in angiocentric glioma drive tumorigenicity through a tripartite mechanism

7 2014, Cancer Discovery, Shern, J. F. et al. Comprehensive Genomic Analysis of Rhabdomyosarcoma Reveals a Landscape of Alterations Affecting a Common Genetic Axis in Fusion-Positive and Fusion-Negative Tumors

8 2019, Nature Communications, Gryder, B. E. et al. Chemical genomics reveals histone deacetylases are required for core regulatory transcription

9 2017, Cancer Discovery, Gryder, B. E. et al. PAX3-FOXO1 Establishes Myogenic Super Enhancers and Confers BET Bromodomain Vulnerability

10 2010, Nat Med, Hettmer, S. \& Wagers, A. J. Muscling in: Uncovering the origins of rhabdomyosarcoma

11 2012, Nature, Dixon, J. R. et al. Topological domains in mammalian genomes identified by analysis of chromatin interactions

12 2014, Cell, Dowen, Jill M. et al. Control of Cell Identity Genes Occurs in Insulated Neighborhoods in Mammalian Chromosomes

13 2015, Cell, Lupiáñez, Darío G. et al. Disruptions of Topological Chromatin Domains Cause Pathogenic Rewiring of Gene-Enhancer Interactions

14 2014, Cell, Rao, Suhas S. P. et al. A 3D Map of the Human Genome at Kilobase Resolution Reveals Principles of Chromatin Looping

15 2017, Genome Biology, Vicente-García, C. et al. Regulatory landscape fusion in rhabdomyosarcoma through interactions between the PAX3 promoter and FOXO1 regulatory elements

16 2016, Nat Meth, Mumbach, M. R. et al. HiChIP: efficient and sensitive analysis of proteindirected genome architecture

17 2014, Cell Reports, Orlando, David A. et al. Quantitative ChIP-Seq Normalization Reveals Global Modulation of the Epigenome

18 2018, Science Translational Medicine, Yohe, M. E. et al. MEK inhibition induces MYOG and remodels super-enhancers in RAS-driven rhabdomyosarcoma

19 2013, Cell, Kadoch, C. \& Crabtree, Gerald R. Reversible Disruption of mSWI/SNF (BAF) Complexes by the SS18-SSX Oncogenic Fusion in Synovial Sarcoma

20 2013, Cell, Whyte, W. A. et al. Master transcription factors and mediator establish superenhancers at key cell identity genes

21 1986, Cell, Hope, I. A. \& Struhl, K. Functional dissection of a eukaryotic transcriptional activator protein, GCN4 of yeast

22 2018, Cell, Boija, A. et al. Transcription Factors Activate Genes through the Phase-Separation Capacity of Their Activation Domains 
23 2014, Nat Genet, Wang, X. et al. Recurrent PAX3-MAML3 fusion in biphenotypic sinonasal sarcoma

24 2017, Genome Res., Siefert, J. C., Georgescu, C., Wren, J. D., Koren, A. \& Sansam, C. L. DNA replication timing during development anticipates transcriptional programs and parallels enhancer activation

25 2013, Cell, Barlow, J. H. et al. Identification of early replicating fragile sites that contribute to genome instability

26 2016, Cell Systems, Durand, N. C. et al. Juicebox Provides a Visualization System for Hi-C Contact Maps with Unlimited Zoom

27 2015, Genome Biology, Servant, N. et al. HiC-Pro: an optimized and flexible pipeline for Hi-C data processing 